\title{
Cordycepin Inhibits Cancer Cell Proliferation and Angiogenesis through a DEK Interaction via ERK Signaling in Cholangiocarcinoma
}

\author{
Tesi Liu, ${ }^{3}$ Guang Zhu, ${ }^{3}$ Wendi Yan, You Lv, Xue Wang, Guang Jin, Minghua Cui, \\ Zhenhua Lin, and Xiangshan Ren
}

Department of Pathology and Cancer Research Center, Yanbian University Medical College, Yanji, China (T.L., G.Z., W.Y., Y.L., X.W., G.J., M.C., Z.L., X.R.); Key Laboratory of the Science and Technology Department of Jilin Province, Yanji, China (T.L., G.Z., W.Y., Y.L., X.W., G.J., M.C., Z.L., X.R.); Key Laboratory of Natural Resources of Changbai Mountain \& Functional Molecules (Yanbian University), Ministry of Education, Yanji, China (T.L., G.Z., W.Y., Y.L., X.W., G.J., M.C., Z.L., X.R.); and Otorhinolaryngology Institute at Otorhinolaryngology Hospital, The First Affiliated Hospital of Sun Yat-sen University, Guangzhou, Guangdong Province, P.R. China (T.L.)

Received October 15, 2019; accepted February 10, 2020

\begin{abstract}
Cholangiocarcinoma (CCA) is a malignant tumor that arises from the epithelial cells of the bile duct and is notorious for its poor prognosis. The clinical outcome remains disappointing, and thus more effective therapeutic options are urgently required. Cordycepin, a traditional Chinese medicine, provides multiple pharmacological strategies in antitumors, but its mechanisms have not been fully elucidated. In this study, we reported that cordycepin inhibited the viability and proliferation capacity of CCA cells in a time- and dose-dependent manner determined by 3-(4,5-dimethylthiazol-2-yl)-2,5-diphenyl tetrazolium bromide (MTT) and colony formation assay. Flow cytometry and Hoechst dye showed that cordycepin induced cancer cell apoptosis via extracellular signal-regulated kinase (ERK) 1/2 deactivation. Moreover, cordycepin significantly reduced the angiogenetic capabilities of CCA in vitro as examined by tube formation assay. We also discovered that cordycepin inhibited DEK expression by using Western blot assay. DEK serves as an oncogenic protein that is overexpressed in various gastrointestinal tumors. DEK silencing inhibited CCA cell viability and angiogenesis but not
\end{abstract}

apoptosis induction determined by Western blot and flow cytometry. Furthermore, cordycepin significantly inhibited tumor growth and angiogenic capacities in a xenograft model by downregulating the expression of DEK, phosphorylated ERK1/ 2 CD31 and von Willebrand factor (vWF). Taken together, we demonstrated that cordycepin inhibited CCA cell proliferation and angiogenesis with a DEK interaction via downregulation in ERK signaling. These data indicate that cordycepin may serve as a novel agent for CCA clinical treatment and prognosis improvement.

\section{SIGNIFICANCE STATEMENT}

Cordycepin provides multiple strategies in antitumors, but its mechanisms are not fully elucidated, especially on cholangiocarcinoma (CCA). We reported that cordycepin inhibited the viability of CCA cells, induced apoptosis via extracellular signalregulated kinase $1 / 2$ deactivation and DEK inhibition, and reduced the angiogenetic capabilities of CCA both in vivo and in vitro.

\section{Introduction}

Cholangiocarcinoma (CCA) is a malignant tumor that originated from the epithelial cells of the bile duct and accounts for $15 \%$ of primary liver cancers (Banales et al., 2016). Because surgery is the only potential curative operation for CCA patients, the clinical outcome remains disappointing,

This study was supported by two National Natural Science Funds of China (31460303, 81660609), Jilin Provincial Science and Technology Department Project (20180101007JC), and Jilin Provincial Education Department Project (No. JJKH20191116KJ).

${ }^{3}$ T.L. and G.Z. contributed equally to this work.

https://doi.org/10.1124/jpet.119.263202. and the survival time is less than 2 years (Blechacz and Gores, 2008). In the last two decades, with the further development of efficient therapeutic strategies, surgical treatment of this disease has become optional. However, CCA is insensitive to radiochemotherapy, and there are serious side effects with combined treatment, thus clinical outcome needs to be improved. Considering the rather unspecific presentation of CCA patients and the aggressiveness of CCA, more specific molecular agents and drugs with low toxicity are urgently required.

Cordycepin, a traditional Chinese medicine extracted from Cordyceps militaris (Fig. 1A), has been reported to exert significant antitumor activities in a wide spectrum of cancers.

ABBREVIATIONS: BM, basal medium; CCA, cholangiocarcinoma; CM, condition medium; DMEM, Dulbecco's modified Eagle's medium; ERK, extracellular signal-regulated kinase; H\&E, hematoxylin and eosin; HUVEC, human umbilical vein endothelial cell; IHC, immunohistochemic; MTT, 3(4,5-dimethylthiazol-2-yl)-2,5-diphenyl tetrazolium bromide; $p$-, phosphorylated; PARP, poly (ADP-ribose) polymerase; PCR, polymerase chain reaction; siRNA, small interfering RNA; VEGF, vascular endothelial growth factor; VEGFR, VEGF receptor; vWF, von Willebrand factor. 
A<smiles>Nc1ncnc2c1ncn2C1OC(CO)C[C@H]1O</smiles>

B
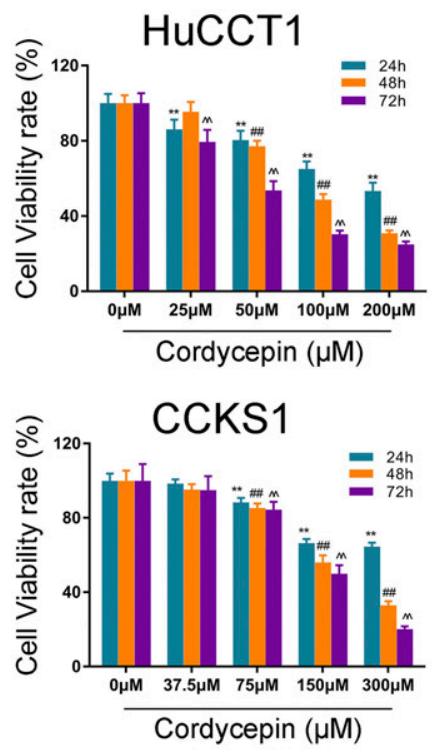

C Cordycepin $(\mu \mathrm{M})$
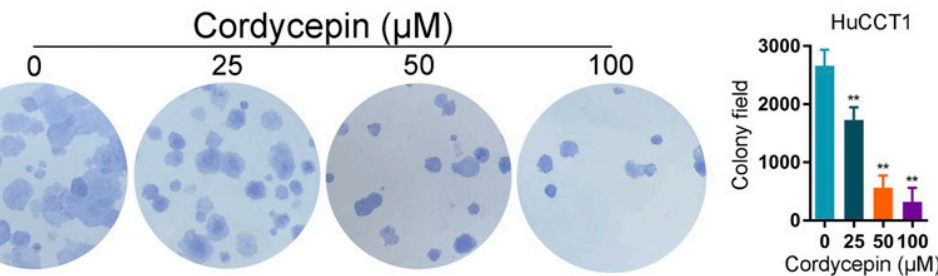

Cordycepin $(\mu \mathrm{M})$

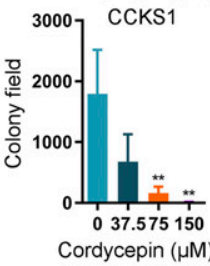

0

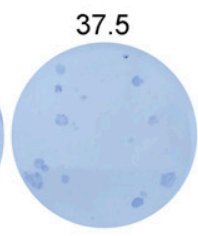

75

150
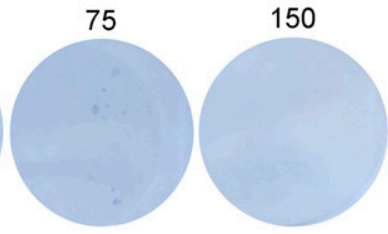

Cordycepin $(\mu \mathrm{M})$

E

HuCCT1
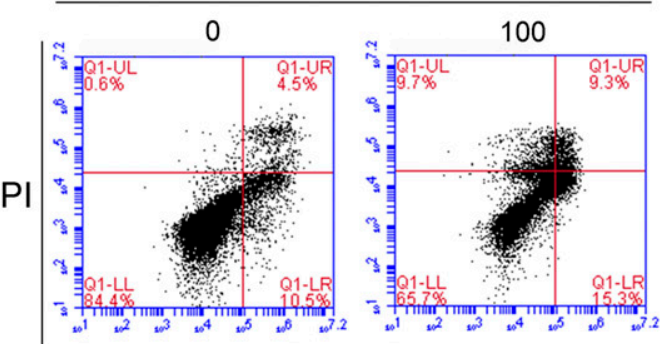

CCKS1

D
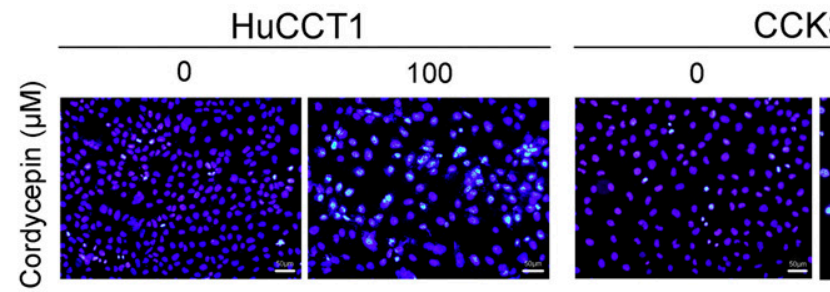

CCKS1

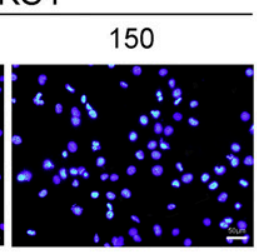

CCKS1

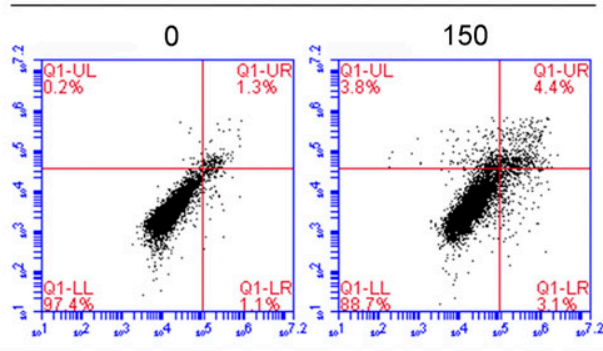

HuCCT1 CCKS1

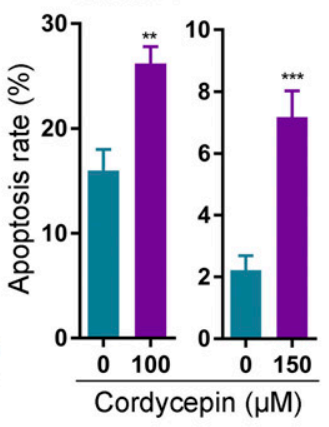

F

HuCCT1

Annexin $\mathrm{V}$

CCKS1

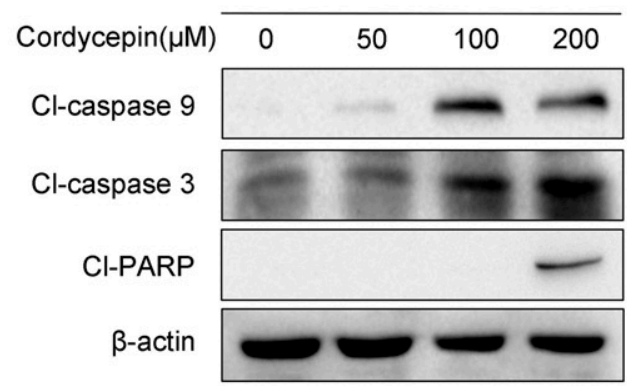

G

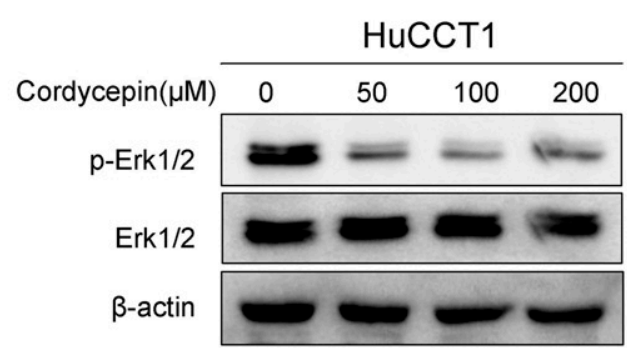

Cl-caspase 3

CI-PARP

$\beta$-actin
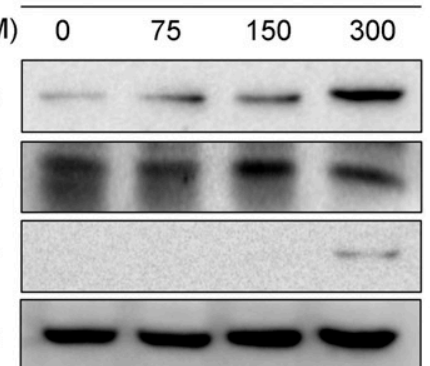

CCKS1

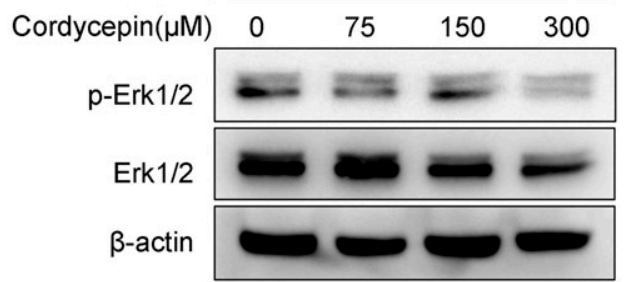

Fig. 1. Cordycepin inhibits CCA cell growth and Erk1/2 phosphorylation. (A) The structure of cordycepin. (B) MTT assay of HuCCT1 and CCKS1 treated with cordycepin at 24-, 48-, and 72-hour time points in a dose-dependent manner; cordycepin significantly inhibited the viability of both cancer cell lines (**P<0.01 vs. 24 hours $0 \mu \mathrm{M}$ group; ${ }^{* \#} P<0.01$ vs. 48 hours $0 \mu \mathrm{M}$ group; ${ }^{\wedge} \wedge P<0.01$ vs. 72 hours $0 \mu \mathrm{M}$ group, $\left.n=6\right)$. (C) Colony formation assay of HuCCT1 and CCKS1 treated with cordycepin in a dose-dependent manner. Cordycepin significantly inhibited the colony formation ability of both cancer 
Cordycepin exerts antiproliferative and antimetastatic and induces apoptosis in cancer cells in vitro (Lee et al., 2010; Jeong et al., 2011). More importantly, cordycepin was reported to have a potential role in RNA/DNA biosynthesis inhibition, antiangiogenesis, and immunomodulation (Lu et al., 2014; Nakamura et al., 2015). Although there are many studies on cordycepin inhibition of cancer, little is known about its molecular mechanisms in CCA.

DEK is an oncogene protein that is overexpressed in aberrant proliferating and malignant cells (RiveiroFalkenbach and Soengas, 2010). Since its first description, DEK has been demonstrated playing roles in DNA repair, replication, and transcription (Soekarman et al., 1992; Kappes et al., 2011). Recent studies showed a pro-oncogenic role of DEK in promoting cell proliferation, survival, and transformation (Wise-Draper et al., 2009; Adams et al., 2015; Privette Vinnedge et al., 2015). DEK functions are wellestablished in cellular processes, but the regulation of DEK is not fully understood. E2 factor, nuclear transcription factor Y, Yin Yang 1, and estrogen receptor $\alpha$ are thought to directly modulate $D E K$ gene transcription (Müller et al., 2001; Privette Vinnedge et al., 2012). DEK was also characterized as a critical regulator of G1/S transition in mammalian cells and was reported play a role in angiogenesis (Zhang et al., 2016). Since DEK is frequently upregulated in various human malignancies and exhibits oncogenic activities, therapeutic strategies that target DEK may form a new approach to cancer treatment.

There are few published reports of the use of cordycepin in CCA treatment, thus we decided to investigate whether cordycepin suppresses CCA progression and to elucidate its molecular mechanisms. MTT and colony formation assays were performed to investigate the impact of cordycepin treatment on cell proliferation, and tube formation assays were conducted to examine its roles in angiogenesis.

In this study, we provide a mechanistic insight into the inhibition of CCA progression and angiogenesis by cordycepin and reveal an interaction between cordycepin and DEK. Our data indicate that cordycepin may serve as a novel therapeutic target for CCA.

\section{Materials and Methods}

Ethics Statement. This research complied with the Helsinki Declaration and was approved by the Human Ethics Committee and the Research Ethics Committee of Yanbian University Medical College. Patients were informed that the resected specimens were stored by the hospital and potentially used for scientific research and their privacy could be maintained.

Cell Culture and Cordycepin Treatment. Human normal biliary epithelial cell line HIBEpic and human CCA cell line QBC939 cells were purchased from American Type Culture Collection (Manassas, VA). The RBE CCA cell line was purchased from Cell Bank of the Type Culture Collection of the Chinese Academy of Sciences (Shanghai, China). CCA cell lines HuCCT1, CCKS1, and TFK1 were provided by the Department of Pathology of Kanazawa University
(Kanazawa, Japan). HIBEpic, QBC939, RBE, HuCCT1, and TFK1 were grown in RPMI-1640 culture medium, and CCKS1 was cultured in Dulbecco's modified Eagle's medium: nutrient Mixture F-12 containing $10 \% \mathrm{FBS}, 1 \%$ glutamine, and $1 \%$ penicillin-streptomycin. Human umbilical vein endothelial cells (HUVECs) were purchased from American Type Culture Collection and grown in endothelial cell growth medium. Cells were maintained in a $37^{\circ} \mathrm{C}$ incubator supplied with $5 \% \mathrm{CO}_{2}$.

For the cordycepin treatment, cordycepin was dissolved in DMSO to a stock solution of $400 \mathrm{mM}$ and further diluted to different concentrations with culture medium. For extracellular signal-regulated kinase (Erk) 1/2 inhibitor GDC-0994 treatment, HuCCT1 cells were treated with 1 and 5 nM GDC-0994 for 48 hours.

Reagents and Antibodies. Cell culture medium (RPMI-1640, DMEM-F12) were purchased from CORNNING. FBS was purchased from BI. For condition medium (CM), cancer cells were seeded after 24 hours and then treated with or without cordycepin for another 48 hours, and the supernatant was collected for tube formation assay (described in the Tube Formation Assay section). For basal medium (BM) used for HUVECs is a medium that can met the basic needs for endothelial cell growth. The BM was used to treat HUVECs without cancer cell preincubation. The purpose of using different media was to avoid the impact on HUVECs caused by the media. Cordycepin was purchased from Sigma Chemical Co. (St Louis, MO). Erk1/2 inhibitor was purchased from ApexBio. The primary antibodies against cleaved caspase 9, cleaved caspase 3, cleaved PARP, p-Erk1/2, and Erk1/2 were purchased from Cell Signaling Technology (Danvers, MA). Vascular endothelial growth factor (VEGF), VEGFR-2, and DEK antibodies were purchased from ProteinTech. CD31 and $\beta$-actin was purchased from Abcam. Von Willebrand factor (vWF) was purchased from Santa Cruz.

MTT Assay. Cells were seeded 5000 cells/well in 96 -well plates and cultured. The cells were then treated with cordycepin at various doses. After 24, 48, and 72 hours, cells were added to MTT solution for 4 hours in the incubator. Then, adding DMSO dissolved MTT and detecting at a full-wavelength spectrophotometer (Tecan, Switzerland).

Colony Formation Assay. Cells were seeded at a density of 2000 cells on six-well plates and cultured. Then cells were treated with cordycepin, respectively. Two weeks later, when colonies were visible, cells were then fixed in formaldehyde $4 \%$, stained by Gimsa $0.5 \%$, and counted manually.

Hoechst33342 Staining. Cells were seeded in six-well plates and cultured until 70\%-80\% confluence. The cells were then treated with cordycepin after 48 hours, fixed in $4 \%$ formaldehydefor, stained for 30 minutes at room temperature with Hoechst33342, and photographed under microscope (SP5II; Leica).

Flow Cytometry Assay. CCA cells were treated with cordycepin after 48 hours, harvested, washed with cold PBS, and resuspended in binding buffer. The cells were stained with annexin $\mathrm{V}$ and/or phosphatidylinositol for 15 minutes, respectively, and this was performed using BD Accuri C6 Software (BD Biosciences, San Jose, CA).

Western Blot Analyses. Cells were collected and lysed with T-PER buffer (Invitrogen) supplemented with protease inhibitors. BCA protein assay (Beyotime Biotechnology, China) was used to measure protein concentration. Proteins were separated using 8\%-10\% SDS-PAGE electrophoresis and transferred to polyvinylidene fluoride membranes. The membranes were blocked and probed with appropriate antibodies. Dilutions of cleaved caspase 9 , cleaved caspase 3, cleaved PARP, p-Erk1/2, Erk1/2, VEGF, VEGFR-2, and

cell lines $(* P<0.05$, **P $<0.01$ vs. $0 \mu \mathrm{M}$ group, $n=3$ ). (D) HuCCT1 and CCKS1 treated with cordycepin for 48 hours, Hoechst33342 dye showed that the apoptotic body was significantly increased (original magnification, $200 \times, n=3$ ). (E) Flow cytometry showed an increase ratio of apoptosis cells after cordycepin treatment of both cell lines with a phosphatidylinositol and annexin $\mathrm{V}$ double staining $(* * P<0.01, * * * P<0.001 \mathrm{vs}$. $0 \mu \mathrm{M}$ group, $n=3$ ). (F) Western blot assay showed a significant increase of Cl-caspase 9, Cl-caspase 3, and Cl-PARP expressions of HuCCT1 and CCKS1 after cordycepin treatment. (G) Western blot assay showed that after cordycepin treatment, p-Erk1/2 expression was significantly downregulated. Cl-, cleaved; PI, phosphatidylinositol. 
A

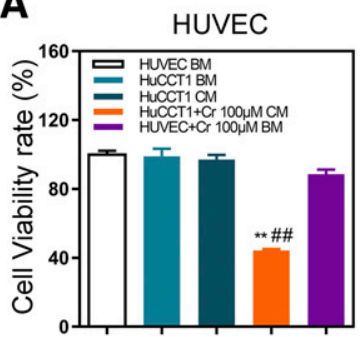

HUVEC

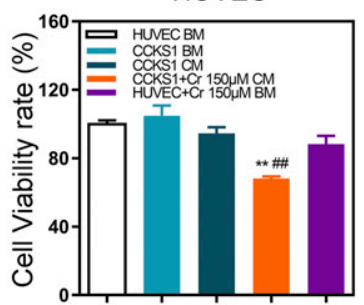

C

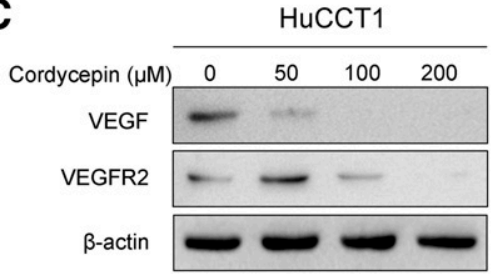

B
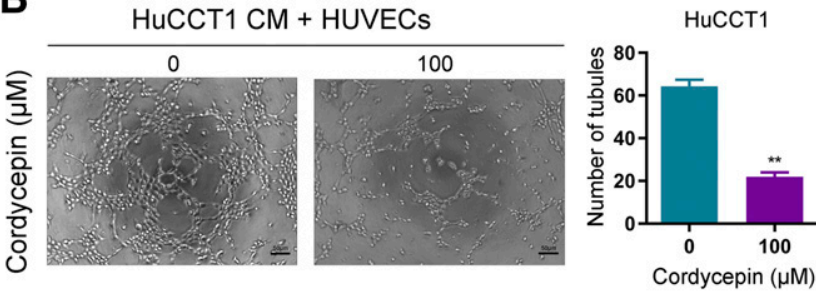

CCKS1 CM + HUVECS
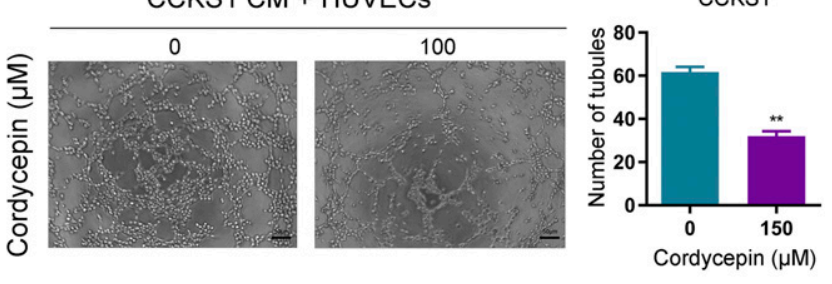

CCKS1

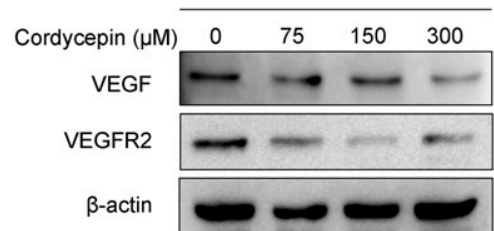

Fig. 2. Cordycepin inhibits tube formation capacities of HUVECs. (A) MTT assay of HUVECs. HUVECs treated with different CM for 48 hours, compared with other groups. CM obtained from both cancer cell lines that were treated with cordycepin (Cr) for 48 hours significantly inhibited HUVECs viability. ( $* * P<0.01$ vs. $0 \mu \mathrm{M}$ group; ${ }^{\# \#} P<0.01$ vs. cordycepin $100 / 150$ $\mu \mathrm{M}$ group, $n=6$ ). (B) HUVECs treated with CM of HuCCT1 and CCKS1. Tube formation assay showed that CM obtained from cancer cells treated with cordycepin significantly inhibited the tube formation ability of HUVECs $(* * P<0.01$ vs. $0 \mu \mathrm{M}$ group, original magnification, $40 \times, n=3$ ). (C) Western blot assay showed that after cordycepin treatment of both cells, expression of VEGF and VEGFR-2 was downregulated.
DEK antibodies were $1: 1000 ; \beta$-actin was $1: 3000$. Plus horseradish peroxidase-labeled secondary antibody was used for incubation, and then electrochemiluminescence color luminescence (Beyotime Biotechnology) was used for detection. $\beta$-actin was used as a loading control.

Tube Formation Assay. Each well of prechilled 96-well plates was bedded with a layer of Matrigel and polymerized at $37^{\circ} \mathrm{C}$ for 1 hour. HUVECs were then resuspended in FBS-free medium with cancer cell culture supernatant. HUVECs were seeded on the polymerized Matrigel and then incubated for 2 hours. The wells were photographed on an inverted phase contrast microscope, and the tube formation ability was evaluated by counting the number of tubes in three random fields.

Small Interfering RNA Transfection. DEK siRNA 4 (5'-TGT CCTCATTAAAGAAGAA- $3^{\prime}$ ) and control siRNA were purchased from RBIOBIO (Guangzhou, China). Cells were transfected with $100 \mathrm{nM}$ siRNA using LipofectAMINE 3000 (Invitrogen) according to the manufacturer's instructions.

Immunohistochemical Staining. A total of 10 human CCA patient specimens collected from 2005 to 2013 were provided by the Affiliated Hospital of Kanazawa University in Japan. Human specimens and xenograft tumor tissues fixed with $4 \%$ paraformaldehydePBS, embedded in paraffin, and sectioned into $4 \mu \mathrm{m}$-thin slices. After antigen retrieval with citric acid $(\mathrm{pH}$ 6.0), endogenous peroxidase activity was blocked with $1 \%$ hydrogen peroxide. Primary antibody p-Erk1/2 antibody (1:200) and DEK antibody (1:250), CD31 antibody (1:100), and vWF (1:200) were applied. CD31 antibody and vWF were used to track the newly formed microvessels. Three optional field were chosen randomly, and the number of vessels was counted. After incubated with secondary antibodies against goat IgG, sections were visualized with diaminobenzidine and counterstained with hematoxylin. The staining was imaged by microscopy.

Reverse-Transcription Polymerase Chain Reaction. The total RNA was extracted using kit (QIAGEN, Germany). cDNA was reversely transcripted from $500 \mathrm{ng}$ of total RNA using reverse transcription kit (TaKaRa, Japan). The cDNA was amplified with the following primers:
5'-AAGGGTGGGATTAAGTACAAGCACA-3' (forward), 5'-ATCCA CGATTGCCTGCACA-3' (reverse) for DEK; and 5'-GAGTCAACGGA TTTGGTCGT-3' (forward), 5'-TTGATTTTGGAGGGATCTC-3' (reverse) for glyceraldehyde-3-phosphate dehydrogenase.

The real-time PCR was carried out with using kit (TaKaRa, Japan). Glyceraldehyde-3-phosphate dehydrogenase was used as an internal control for each sample.

Xenograft Model. This study was approved by the ethics committee of the Yanbian University Medical College. Four-week-old male nude mice were obtained from Hua Fu Kang bioscience company Beijin, China, and HuCCT1 $\left(5 \times 10^{6}\right.$ cells/mouse $)$ in $100 \mu$ l of FBS-free medium was subcutaneously injected. Tumor nodules were examined every day and were evaluated using the following formula: tumor volume $=\left(\right.$ width $^{2} \times$ length $) / 2$. Seven days after tumor cell inoculation, 10 mice with different tumor volumes were divided randomly into two groups (five animals per group). The treatment group received cordycepin treatment via intraperitoneal injection every day $(50 \mathrm{mg} / \mathrm{kg}$ ), and the control group received the same volume of PBS. The body weight and tumor volume of each mouse were assessed every 2 days using caliper measurements. On day 45 , the mice were sacrificed, and the livers and tumors were excised and fixed for immunohistochemistry staining and hematoxylin and eosin (H\&E) staining. This experiment was accomplished in Changchun Shengyu cell biotechnology co. LTD, China.

Statistics. Statistical analysis was conducted by SPSS 17.0 software (SPSS Inc.) and GraphPad Prism version 5.0 (GraphPad, San Diego, CA). Measurements were indicated as mean \pm S.D. and evaluated using Student's $t$ test and one-way ANOVA. A $P$ value $<0.05$ was used as a measure of statistically significance.

\section{Results}

Cordycepin Inhibits Cancer Cell Growth and Erk1/2 Phosphorylation in CCA. Cordycepin is reported to exert significant antitumor activities (Yang et al., 2017a; Zhou et al., 2017; Chaicharoenaudomrung et al., 2018). To investigate 
A

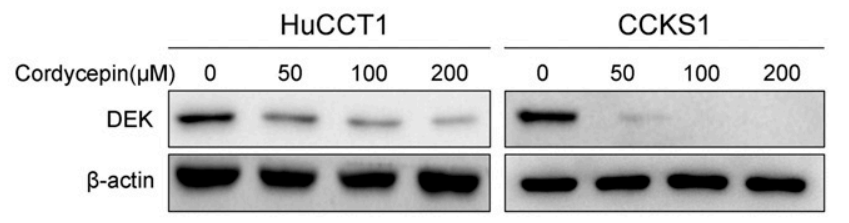

C
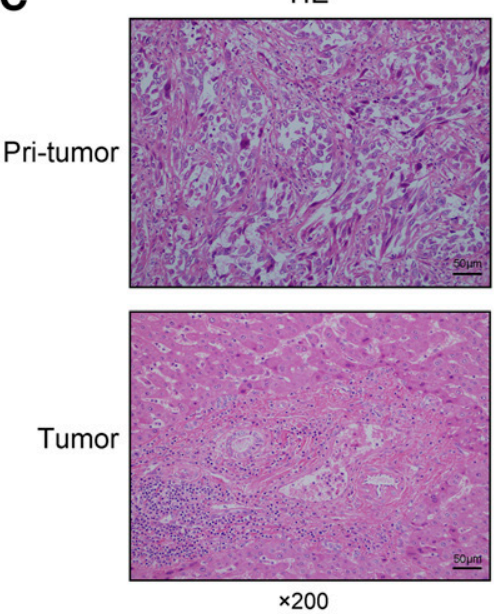

$\times 200$

D

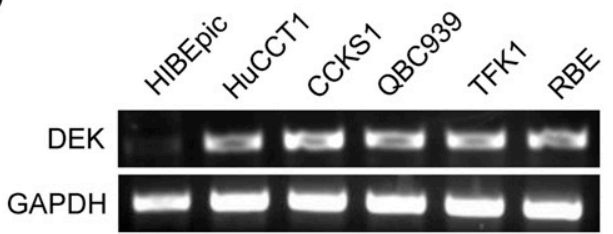

whether cordycepin influences the progression of CCA, HuCCT1 and CCKS1 cells were treated with cordycepin in a time- and dose-dependent manner. Different doses of cordycepin used for cancer cell treatment was based on the different $\mathrm{IC}_{50}$ value of the cell, as shown in Fig. $1 \mathrm{~B}$. The $\mathrm{IC}_{50}$ value of HuCCT1 was $100 \mu \mathrm{M}$ and the value for CCKS1 was $150 \mu \mathrm{M}$. Cell viability was determined by MTT assay. Cordycepin significantly reduced cell viability in both HuCCT1 and CCKS1 cells (Fig. 1B). The colony formation ability of both HuCCT1 and CCKS1 was reduced in a dosedependent manner after cordycepin treatment of 2 weeks (Fig. 1C). These results demonstrated that cordycepin inhibits CCA progression by inhibiting CCA cell proliferation. Because mounting data has demonstrated that cordycepin provides multiple pharmacological strategies for apoptosis induction in various kinds of cancers, we examined whether cordycepin exerts apoptosis induction in both CCA cell lines. We found that after 48 hours of treatment with cordycepin in both HuCCT1 and CCKS1 cell lines, Hoechst33342 staining revealed a significant increase in cell apoptosis (Fig. 1D). Quantitative measurement of annexin $\mathrm{V}$ and propidium iodide positivity was performed by flow cytometry. Both cell lines showed a significant increase in apoptosis after 48 hours of cordycepin treatment (Fig. 1E). We then analyzed the expression of apoptosis markers by Western blot assay. Cordycepin treatment resulted in a significant increase in cleaved caspase-9, cleaved caspase-3, and cleaved PARP (Fig. 1F).
B TCGA

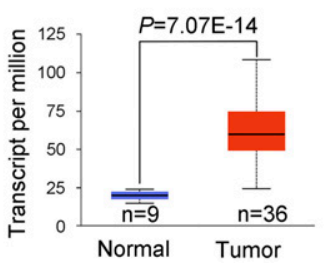

DEK
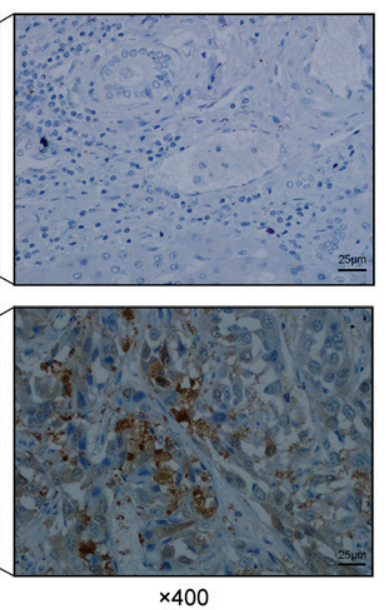

Fig. 3. Cordycepin downregulated DEK overexpression in CCA. (A) Western blot assay showed that DEK expression in HuCCT1 and CCKS1 was downregulated after cordycepin treatment. (B) UALCAN data base based on The Cancer Genome Atlas data showed that DEK is overexpressed in CCA. (C) IHC staining of DEK. DEK showed a strongly positive staining in CCA tissue. (D) Reverse-transcription PCR assay showed that DEK is overexpressed in five different CCA cell lines. Abbreviations: HE, hematoxylin and eosin; Pri-, peripheral. UALCAN data base: http://ualcan.path.uab.edu.
Previous reports showed that the ERK pathway is involved in tumor initiation and progression, and a reduction in ERK activation resulted in CCA growth inhibition (Chen et al., 2018; Yokoi et al., 2018). Thus, Western blot assays were performed to evaluate ERK expression. Protein expression was evaluated following treatment of both cell lines with various doses of cordycepin for 24 hours. In both cancer cell lines, p-ERK1/2 levels were significantly decreased (Fig. 1G). These results demonstrated that cordycepin attenuates CCA cell proliferation by apoptosis induction and ERK1/2 inhibition.

Cordycepin Inhibits the Tube Formation Capacities of HUVECs. Earlier studies indicated that cordycepin has an antiangiogenetic role in cancer suppression (Lu et al., 2014). Given that angiogenesis is essential for cancer progression and metastasis, we employed tube formation assays in HUVECs to determine whether cordycepin influences CCA angiogenesis. First, to abolish the influence of cell viability of HUVECs in tube formation assays and to further elucidate how cordycepin inhibits CCA angiogenesis, MTT assays were performed. HUVECs were incubated in DMEM, HUVEC BM, RPMI-1640/DMEM-F12 cancer cell culture BM, RPMI-1640/ DMEM-F12 plus 100/150 $\mu \mathrm{M}$ cordycepin, CM from both cancer cell lines, and the $\mathrm{CM}$ from both cancer cell lines after 48 hours cordycepin treatment. MTT assays revealed that only incubation with CM obtained from both cancer cell lines after 48 hours cordycepin treatment significantly reduced the 
viability of HUVECs. We speculated that cordycepin may inhibit CCA angiogenesis and that the CM obtained from cancer cell lines after cordycepin treatment may contain some apoptotic factors that further influence HUVEC cell viability (Fig. 2A). Next, we incubated HUVECs with the CM obtained from both cancer cell lines after 48 hours of cordycepin treatment. We found that the tube formation ability of HUVECs was attenuated in the cordycepin-treated group in both cell lines (Fig. 2B). We then assessed the expression of VEGF and VEGFR-2 by Western blotting. Cordycepin treatment lowered VEGF and VEGFR-2 expression, which further confirmed the tube formation results (Fig. 2C). Our results indicated that cordycepin slowed CCA progression by angiogenesis inhibition.

Cordycepin Downregulated DEK Overexpression in CCA Cell Lines. DEK is reported as an oncogene in many types of cancers, like acute myeloid leukemia, bladder cancer, hepatocellular cancer, and breast cancer (Liu et al., 2017). Recent reports showed that DEK interact with lung cancer metastasis-related protein 1 to enhance cancer cell growth and suppress apoptosis (Xu et al., 2017). A previous study also showed that DEK has critical roles in angiogenesis and phosphoinositide 3-kinase/protein kinase $\mathrm{B} /$ mammalian target of rapamycin pathway modulation (Yang et al., 2017b). Considering these findings, and compared with our previous studies of cordycepin, we hypothesized that cordycepin may influence DEK expression. Evaluation of DEK expression in both cancer cell lines was undertaken by Western blotting. We found an inverse relationship between cordycepin and DEK expression, as shown in Fig. 3A; DEK demonstrated significantly lower expression after cordycepin treatment. DEK expression in various cancer types was also confirmed. Data obtained from the UALCAN data base (UALCAN data base: http://ualcan.path.uab.edu) showed that DEK is overexpressed in CCA (Fig. 3B). To further confirm our results, IHC staining was undertaken. Compared with peri-tumor tissue, DEK showed strongly positive staining in CCA tumor tissue (Fig. 3C). We then analyzed $D E K$ mRNA expression
A

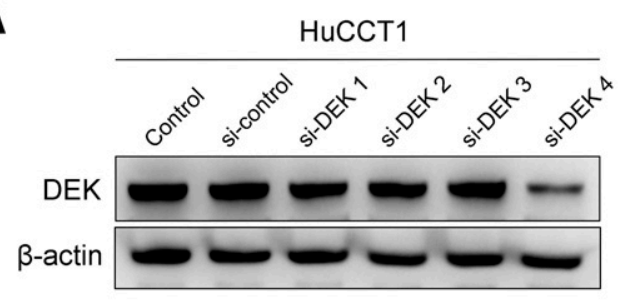

C

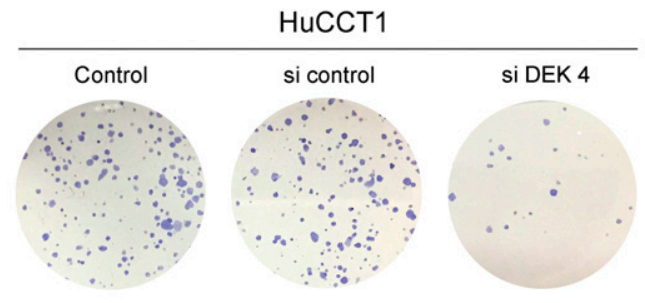

D

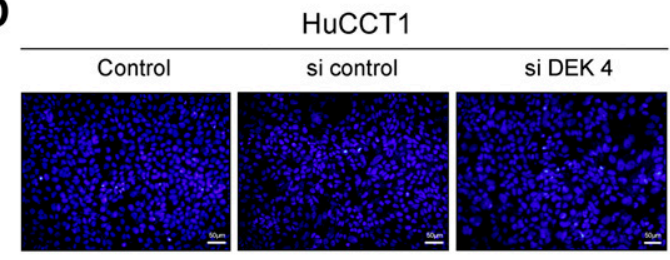

E

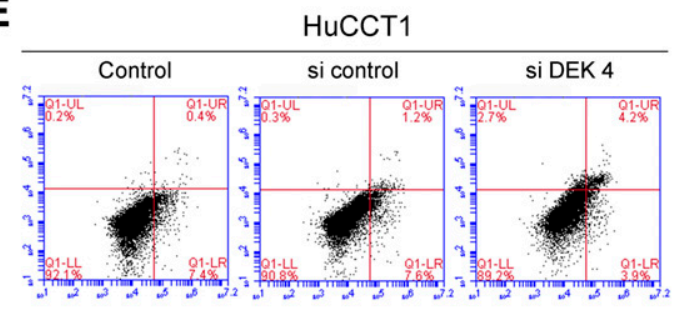

B
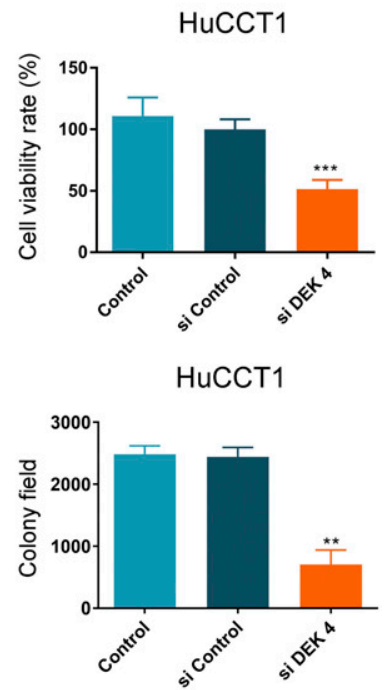

$\mathbf{F}$

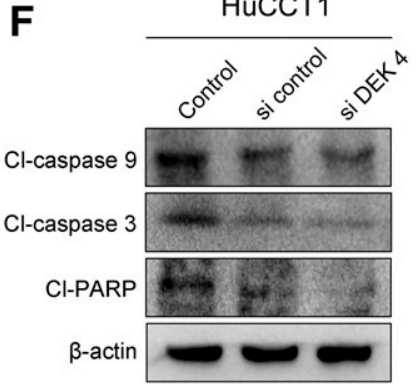

HuCCT1

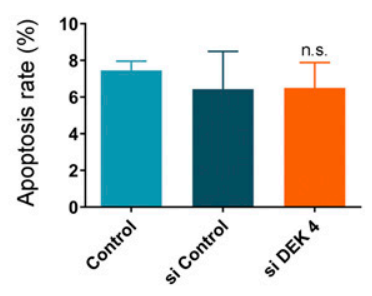

Fig. 4. Silencing DEK attenuates CCA cell growth. (A) Western blot assay to demonstrate the knockdown significance of si DEK. si, short interfer. (B) MTT assay showed that the cell viability of HuCCT1 were downregulated by si DEK $4{ }^{* * * *} P<0.001$ vs. si Control group, $n=$ 6). (C) Colony formation assay of HuCCT1. The ability of colony formation of HuCCT1 was inhibited by si DEK $4(* * P<0.01$ vs. si Control group, $n=3$ ). (D) Hoechst33342 dye showed no significant changes in apoptosis induction in HuCCT1 after silencing DEK. $(n=3)$. (E) Flow cytometry showed no significant changes in apoptosis induction after silencing DEK. ( $n=$ 3). (F) Western blot assay showed no significant changes in expression of Cl-caspase 9, Clcaspase 3, and Cl-PARP. Cl-, cleaved; n.s., not significant. 
A

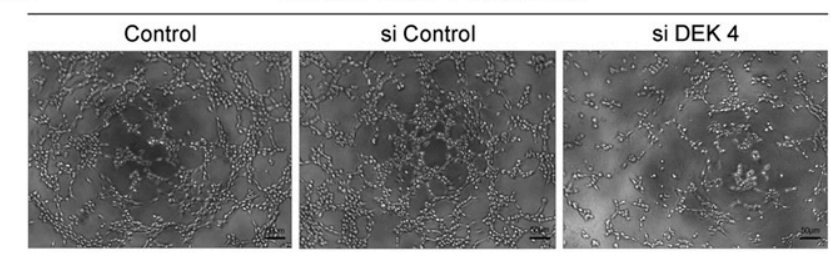

B

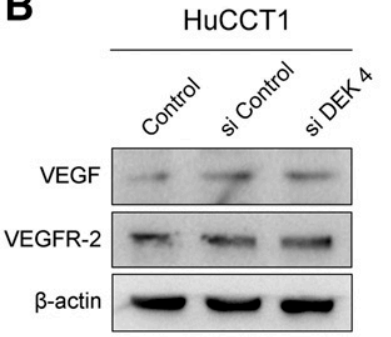

E C HUVEC

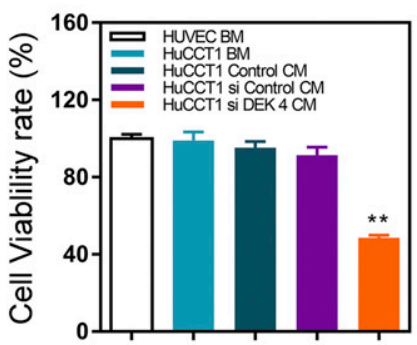

HuCCT1

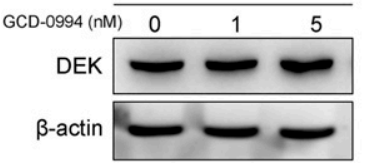

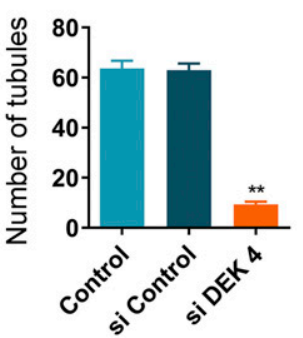

D

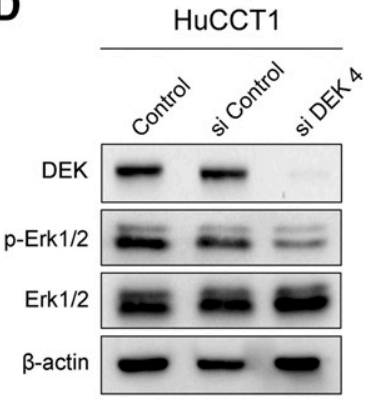

Fig. 5. DEK silencing attenuated tube formation capacities of HUVECs and inhibited Erk1/2 signaling. (A) Tube formation assay of HUVECs. The ability of tube formation was inhibited after HUVECs were treated with the culture supernatant obtained from DEK-silencing cancer cell (** $P<0.01$ vs. si Control group, original magnification, $40 \times, n=3$ ). (B) Western blot assay showed no significant changes in VEGF and VEGFR-2 expression. (C) MTT assay of HUVECs. HUVECs treated with different culture supernatant for 48 hours, compared with other groups. Culture supernatant obtained from DEK-silencing HuCCT1 significantly inhibited HUVECs viability. ( $* * P<0.01$ vs. $0 \mu \mathrm{M}$ group, $n=3$ ). (D) Western blot assay showed that the expression of $\mathrm{p}$-Erk $1 / 2$ was downregulated by silencing DEK. (E) Western blot assay showed no significant changes in DEK expression after GDC-0994 treatment. Abbreviations: si, short interfer. levels by reverse-transcription PCR in HIBEpic cells, a normal epithelial cell line of the bile duct, and five CCA cell lines: HuCCT1, CCKS1, QBC939, TFK1, and RBE. As shown in Fig. 3D, the expression levels of DEK in five cancer cell lines were much higher than those in HIBEpic cells. Together, these data imply that DEK is overexpressed in CCA cell lines, and cordycepin suppression of DEK expression may contribute to its roles in CCA inhibition.

DEK Silencing Inhibits CCA Growth. To further confirm the oncogenic roles of DEK in CCA and the correlation between cordycepin and DEK, we next silenced $D E K$ expression using siRNA. Western blotting analysis enabled selection of siRNA sequence 4 for further investigation (Fig. 4A). MTT assay showed a significant decrease in cell viability in HuCCT1 cells after DEK silencing (Fig. 4B), and colony formation assays also revealed a reduction in colony formation capacity (Fig. 4C). To gain a mechanistic understanding of the potential role of DEK in modulating tumorigenesis, flow cytometry and Hoechst33342 staining were used to investigate the roles of DEK in apoptosis. As shown in Fig. 4, D and E, no significant changes were observed in apoptosis induction, and Western blotting also confirmed no changes in apoptosis markers (Fig. 4F). Herein, we indicate that DEK increased tumor cell growth by enhancing its proliferation rate, and $D E K$ knockdown is irrelevant to apoptosis induction.

DEK Silencing Attenuated the Tube Formation Capacities of HUVECs and Inhibited Erk1/2 Signaling. We next analyzed the role of DEK in angiogenesis, and tube formation assays revealed that $D E K$ silencing significantly inhibited the ability of HUVECs to form tubes (Fig. 5A). Intriguingly, VEGF and VEGFR-2 demonstrated no significant changes in expression (Fig. 5B). Therefore, further investigation is required. MTT assays showed that, compared with the other four groups, HUVECs incubated with CM obtained from $D E K$-silenced HuCCT1 cells underwent viability attenuation (Fig. 5C). Previous studies demonstrated that DEK has critical roles in tumorigenesis via ERK1/2 modulation, hence Western blot assays were used to analyze p-ERK1/ 2 expression after $D E K$ silencing. Compared with the control group, $D E K$ silencing inhibited the phosphorylation of ERK1/2 (Fig. 5D). We next applied GDC-0994, an ERK1/2 inhibitor to further confirm the role of DEK in ERK1/2 activation. Western blot assay showed that there were no significant changes in DEK expression (Fig. 5E), suggesting that DEK exerts oncogenic functions via ERK1/2 modulation.

Cordycepin Inhibits CCA Progression by Targeting DEK via ERK1/2 Signaling in a Xenograft Model. To further confirm the antitumor effects of cordycepin in vivo, $5 \times$ $10^{6}$ HuCCT1 cells were subcutaneously transplanted into nude mice ( $n=5$ biologically independent mice per group). Seven days after cancer cell injection, cordycepin at a dose of $50 \mathrm{mg} / \mathrm{kg}$ was administered every 24 hours for 45 days by intraperitoneal injection, and then the mice were killed, and the transplanted tumors were excised (Fig. 6A). There were no significant changes in body weight (Fig. 6B), and tumor volumes underwent a significant reduction compared with the control group (Fig. 6C). We then evaluated the subcutaneously transplanted tumors at the end of experiment; after cordycepin treatment, tumor weight and tumor volume were both reduced (Fig. 6, D and E). It was also obvious that there were far fewer microvessels on the tumor surface compared with the control group and indicated that angiogenesis was inhibited by cordycepin (Fig. 6F). H\&E staining of the portal tract was undertaken to evaluate the toxicity of cordycepin and revealed no significant changes (Fig. 6G), indicating that little damage was done by cordycepin at a dose of $50 \mathrm{mg} / \mathrm{kg}$. 
A

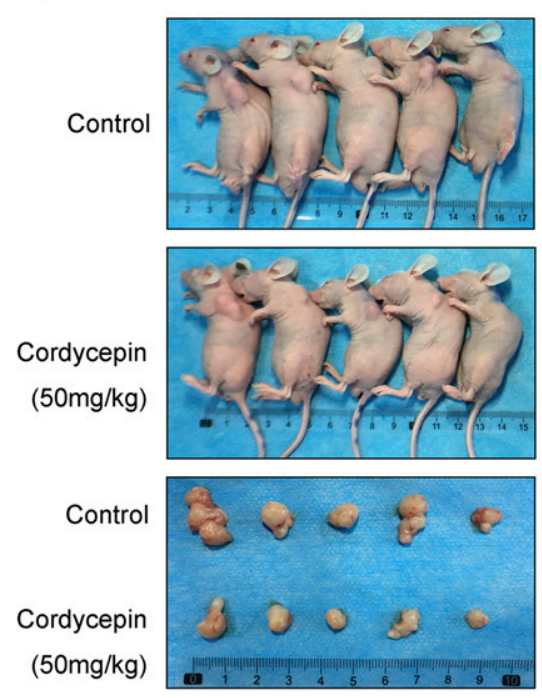

B

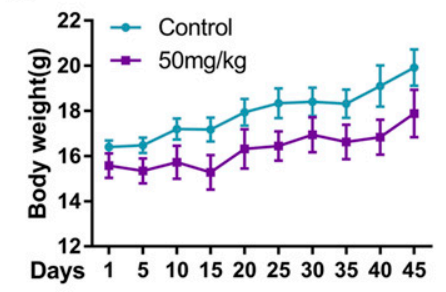

C

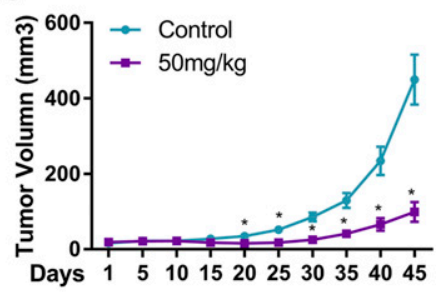

$F$

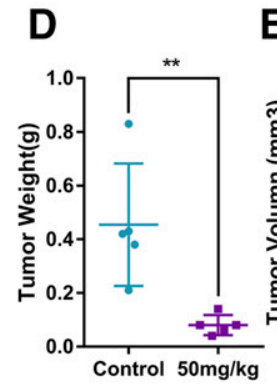

E

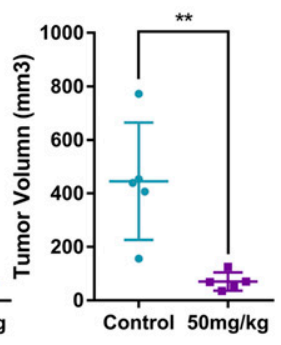

\section{Control $\quad 50 \mathrm{mg} / \mathrm{kg}$}
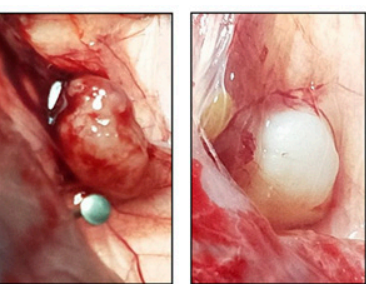

\section{G}
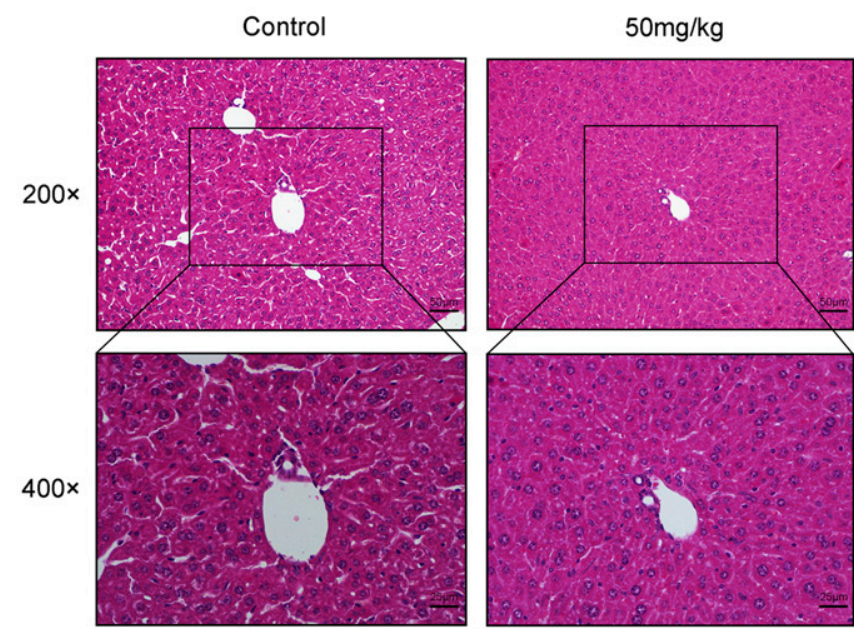

Fig. 6. Cordycepin inhibits CCA growth via DEK and p-Erk1/2 inhibition in vivo. (A) Representative images of nude mice xenografted with HuCCT1 cells and treated with or without cordycepin $50 \mathrm{mg} / \mathrm{kg}$. (B) Changes of body weight after 45 days of cordycepin treatment. No significant changes were found. (C) Changes of tumor volume after 45 days of cordycepin treatment. Cordycepin treatment significantly inhibited the tumor growth of HuCCT1 in a xenograft model ( $* P<0.05, * * P<0.01 \mathrm{vs.} \mathrm{Control} \mathrm{group).} \mathrm{(D)} \mathrm{Comparison} \mathrm{of} \mathrm{tumor} \mathrm{weight} \mathrm{and} \mathrm{cordycepin} \mathrm{efficacy.} \mathrm{(E)} \mathrm{Comparison} \mathrm{of} \mathrm{tumor} \mathrm{volume}$ and cordycepin efficacy. (F) Pictures of xenograft tumor after cordycepin treatment. Compared with control group, the tumor volume was much smaller after cordycepin treatment and few micro-blood vessels were found. (G) H\&E staining of the portal tract to evaluate the toxicity of cordycepin. No significant changes were found. (H) IHC and H\&E staining of the xenograft tumor tissue. IHC staining showed a significant inhibition of p-Erk1/2 and DEK expression. (I) The IHC staining of two angiogenic markers CD31 and vWF showed that the newly formed endothelial cells and microvessels (marked by red arrowhead) were significantly reduced after cordycepin treatment. HE, hematoxylin and eosin.

Next, we applied IHC and H\&E staining of the xenograft tumor tissue. IHC staining showed a significant inhibition of p-ERK1/2 and DEK expression compared with the control group (Fig. $6 \mathrm{H}$ ). The IHC staining of two angiogenic markers CD31 and vWF showed that the newly formed endothelial cells and microvessels were significantly reduced after cordycepin treatment (Fig. 6I). Thus, our data indicated that cordycepin inhibited CCA progression by targeting angiogenic capacities and DEK via ERK1/2 signaling in vivo.

\section{Discussion}

Multiple studies have shown that cordycepin exerts antitumor effects in hepatocellular carcinoma, colorectal cancer, and gastric cancer (He et al., 2010; Nasser et al., 2017; Zeng et al.,
2017). Researches have indicated that cordycepin suppresses tumor growth by apoptosis induction and angiogenesis inhibition. However, in CCA, the effects of cordycepin were not fully elucidated. Therefore, we performed MTT and colony formation assays to investigate the impact of cordycepin treatment on cell proliferation. MTT assays revealed attenuated cell viability and colony formation ability in both CCA cell lines. Further investigations utilizing Hoechst dye and flow cytometry revealed apoptosis induction in both cell lines following cordycepin treatment. Parallel with Western blot results, the apoptosis markers, cleaved caspase-3, cleaved caspase-9, and cleaved PARP, were upregulated, which further confirmed the apoptosis induction effects of cordycepin. Previous studies have suggested that cordycepin induces apoptotic effects by downregulating ERK signaling (Wang 
H
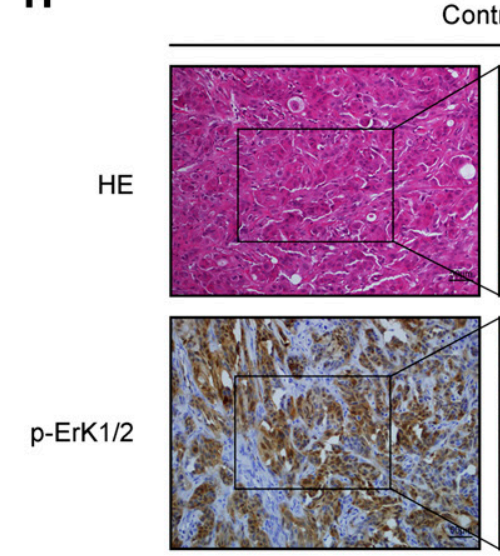

DEK

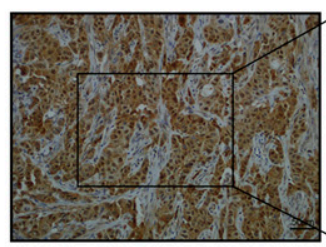

$200 x$
Control
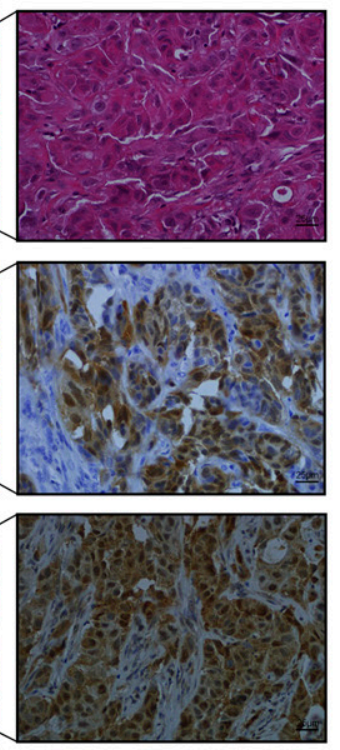

$400 x$

I
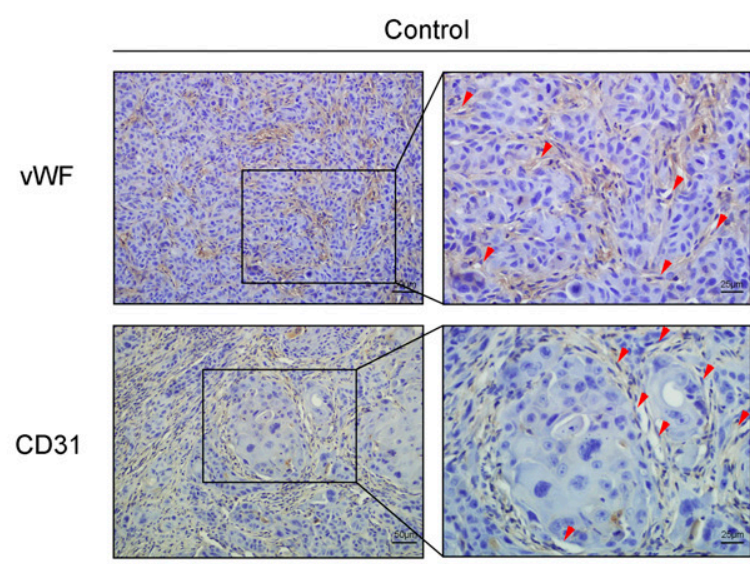

$200 x$

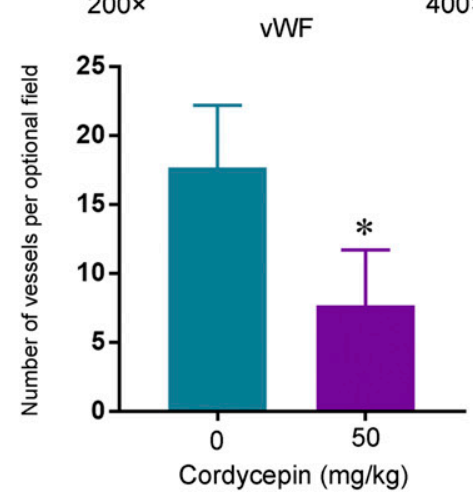

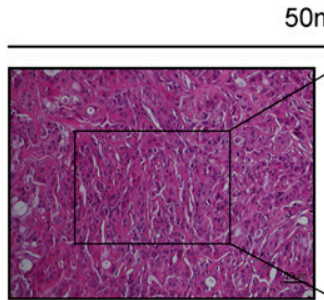

$50 \mathrm{mg} / \mathrm{kg}$
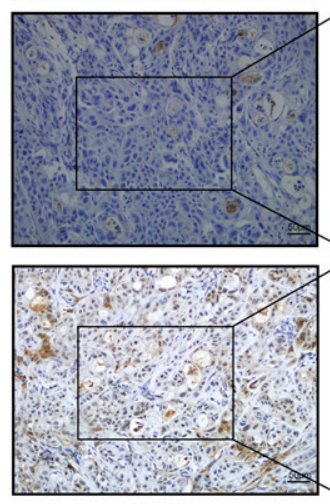

$200 x$
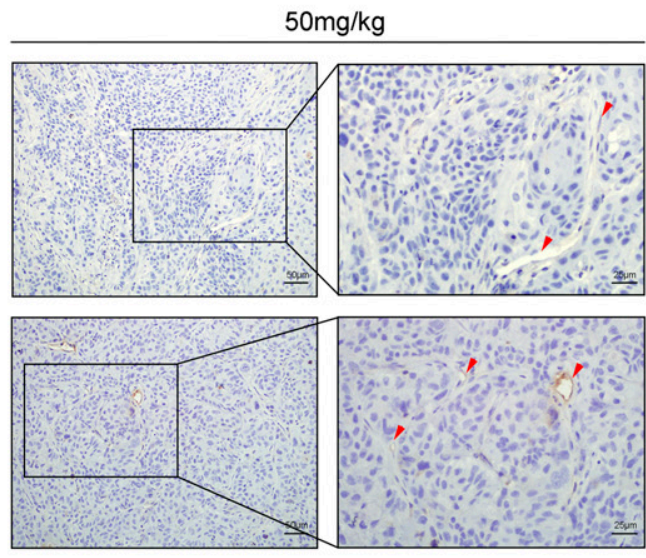

CD31

$400 x$

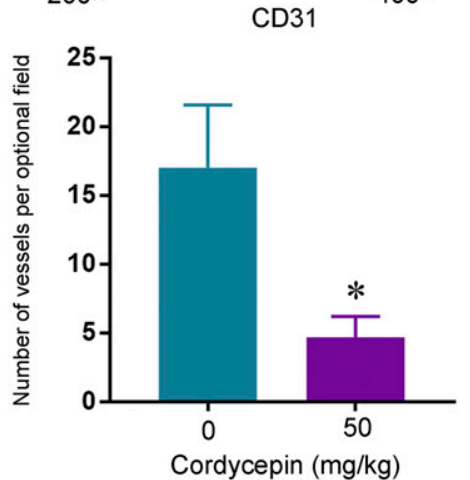

Fig. 6. Continued.

et al., 2017), and ERK1/2 activation promotes CCA cell proliferation, chemoresistance, and progression (Ewald et al., 2014; Yokoi et al., 2018). Thus, analysis of p-ERK1/2 expression after cordycepin treatment was performed. Compared with the control group, cordycepin treatment significantly inhibited ERK1/2 phosphorylation, suggesting that cordycepin inhibits CCA cell proliferation dependent upon ERK1/2 downregulation.

Angiogenesis, another aspect that promotes cancer progression, was also investigated in this study. Given that angiogenesis provides various benefits and comforts microenvironment for tumor growth (Okkenhaug et al., 2016; Xiang 


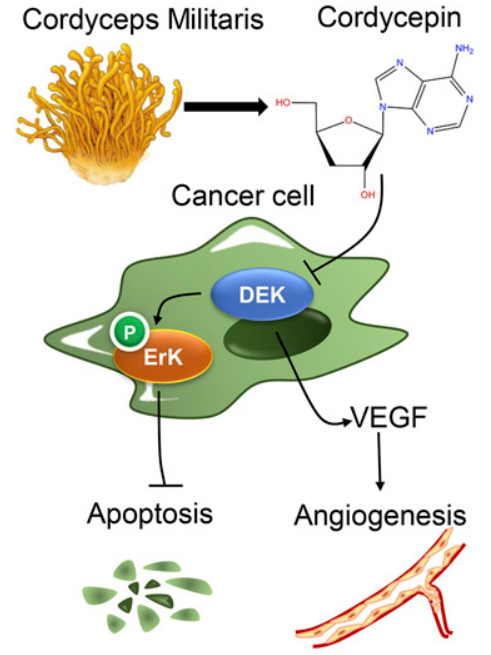

Fig. 7. Schematic representation of the molecular mechanism of cordycepin. Schematic representation of the molecular mechanism of cordycepin targeting DEK, which inhibits cancer cells proliferation by apoptosis induction and angiogenesis inhibition.

et al., 2017; Yang et al., 2018), CCA progression also takes the advantages (Kangsamaksin et al., 2017; Xu et al., 2019). Indeed, tube formation assay, which was performed by HUVECs in vitro, revealed an association between angiogenesis and cordycepin antitumor functions. In our data, cordycepin inhibited HUVECs tube formation ability in vitro, and angiogenesis-associated protein, VEGF, and VEGFR-2 were also downregulated. We therefore believe cordycepin may play an antitumor role by targeting angiogenesis in CCA progression.

Cordycepin have been demonstrated targeting various oncogenes to achieve antitumor effects (Liang et al., 2017; Zeng et al., 2017; Cui et al., 2018). DEK acts as an oncogene and is overexpressed in various cancer types, including CCA. DEK was proven to enhance tumorigenesis and tumorassociated angiogenesis. In this study, notably, we found DEK expression was downregulated by cordycepin treatment. To further elucidate the roles of cordycepin and DEK interplay, we silenced DEK using siRNA and examined its roles in tumor growth and angiogenesis. According to our studies, DEK silencing significantly reduced cell viability and colony formation capacities. However, DEK silencing was found to be irrelevant with apoptosis induction in CCA. In contrast, DEK silencing was found to promote apoptosis in cervical cancer and lung cancer (Liu et al., 2012; Feng et al., 2017); we speculated that cordycepin-modulated cell death may mainly be due to Erk1/2 deactivation but not DEK silencing. DEK silencing revealed no changes in cell apoptosis induction, which may be due to cancer specificity, and further studies are required.

Finally, DEK silencing was also associated with tube formation in HUVECs and downregulation of ERK1/2 signaling. These data revealed that cordycepin functions correspond with DEK silencing and that DEK may act as a potential target of cordycepin. We next generated a xenograft model to further confirm our findings. Cordycepin treatment significantly downregulated the relative volume of subcutaneously transplanted tumors, and IHC staining of tumor tissue revealed that p-ERK1/2 and DEK were inhibited. In conclusion, our study provides mechanistic insights into the inhibition of CCA progression and angiogenesis by cordycepin and revealed an interaction with DEK (Fig. 7).

Our data indicate that cordycepin may serve as a novel therapeutic target for CCA.

\section{Acknowledgments}

The authors would like to thank Dr. Kenichi Harada and Dr. Yasunori Sato (Department of Human Pathology, Kanazawa University Graduate School of Medicine, Kanazawa, Japan) for their valuable suggestions for this experiment design and their technical support during the preparation of this manuscript.

\section{Authorship Contributions}

Participated in research design: Lin, Ren

Conducted experiments: Liu, Zhu, Yan, Lv, Wang.

Patient sample collection: Jin, Cui.

Data analysis: Liu, Zhu.

Wrote or contributed to the writing of the manuscript: Liu, Zhu.

\section{References}

Adams AK, Hallenbeck GE, Casper KA, Patil YJ, Wilson KM, Kimple RJ, Lambert PF, Witte DP, Xiao W, Gillison ML, et al.(2015) DEK promotes HPV-positive and -negative head and neck cancer cell proliferation. Oncogene 34:868-877.

Banales JM, Cardinale V, Carpino G, Marzioni M, Andersen JB, Invernizzi P, Lind GE, Folseraas T, Forbes SJ, Fouassier L, et al.(2016) Expert consensus document: cholangiocarcinoma: current knowledge and future perspectives consensus statement from the European Network for the Study of Cholangiocarcinoma (ENSCCA). Nat Rev Gastroenterol Hepatol 13:261-280.

Blechacz B and Gores GJ(2008) Cholangiocarcinoma: advances in pathogenesis, diagnosis, and treatment. Hepatology 48:308-321.

Chaicharoenaudomrung N, Jaroonwitchawan T, and Noisa $\mathrm{P}(2018)$ Cordycepin induces apoptotic cell death of human brain cancer through the modulation of autophagy. Toxicol In Vitro 46:113-121.

Chen J, Song W, Du Y, Li Z, Xuan Z, Zhao L, Chen J, Zhao Y, Tuo B, Zheng S, et al.(2018) Inhibition of KLHL21 prevents cholangiocarcinoma progression through regulating cell proliferation and motility, arresting cell cycle and reducing Erk activation. Biochem Biophys Res Commun 499:433-440.

Cui ZY, Park SJ, Jo E, Hwang IH, Lee KB, Kim SW, Kim DJ, Joo JC, Hong SH, Lee MG, et al.(2018) Cordycepin induces apoptosis of human ovarian cancer cells by inhibiting CCL5-mediated Akt/NF-кB signaling pathway. Cell Death Discov 4:62.

Ewald F, Nörz D, Grottke A, Hofmann BT, Nashan B, and Jücker M(2014) Dual inhibition of PI3K-AKT-mTOR- and RAF-MEK-ERK-signaling is synergistic in cholangiocarcinoma and reverses acquired resistance to MEK-inhibitors. Invest New Drugs 32:1144-1154.

Feng T, Liu Y, Li C, Li Z, and Cai H(2017) DEK proto-oncogene is highly expressed in astrocytic tumors and regulates glioblastoma cell proliferation and apoptosis. Tumour Biol 39:1010428317716248.

He W, Zhang MF, Ye J, Jiang TT, Fang X, and Song Y(2010) Cordycepin induces apoptosis by enhancing JNK and p38 kinase activity and increasing the protein expression of Bcl-2 pro-apoptotic molecules. J Zhejiang Univ Sci B 11:654-660.

Jeong JW, Jin CY, Park C, Hong SH, Kim GY, Jeong YK, Lee JD, Yoo YH, and Choi

$\mathrm{YH}(2011)$ Induction of apoptosis by cordycepin via reactive oxygen species generation in human leukemia cells. Toxicol In Vitro 25:817-824.

Kangsamaksin T, Chaithongyot S, Wootthichairangsan C, Hanchaina R, Tangshewinsirikul C, and Svasti J(2017) Lupeol and stigmasterol suppress tumor angiogenesis and inhibit cholangiocarcinoma growth in mice via downregulation of tumor necrosis factor- $\alpha$. PLoS One 12:e0189628.

Kappes F, Khodadoust MS, Yu L, Kim DS, Fullen DR, Markovitz DM, and Ma L(2011) DEK expression in melanocytic lesions. Hum Pathol 42:932-938.

Lee SJ, Moon GS, Jung KH, Kim WJ, and Moon SK(2010) c-Jun N-terminal kinase 1 is required for cordycepin-mediated induction of G2/M cell-cycle arrest via p21WAF1 expression in human colon cancer cells. Food Chem Toxicol 48:277-283.

Liang SM, Lu YJ, Ko BS, Jan YJ, Shyue SK, Yet SF, and Liou JY(2017) Cordycepin disrupts leukemia association with mesenchymal stromal cells and eliminates leukemia stem cell activity. Sci Rep 7:43930.

Liu G, Xiong D, Zeng J, Xu G, Xiao R, Chen B, and Huang Z(2017) Prognostic role of DEK in human solid tumors: a meta-analysis. Oncotarget 8:98985-98992.

Liu K, Feng T, Liu J, Zhong M, and Zhang S(2012) Silencing of the DEK gene induces apoptosis and senescence in CaSki cervical carcinoma cells via the up-regulation of NF-кB p65. Biosci Rep 32:323-332.

Lu H, Li X, Zhang J, Shi H, Zhu X, and He X(2014) Effects of cordycepin on HepG2 and EA.hy926 cells: potential antiproliferative, antimetastatic and anti-angiogenic effects on hepatocellular carcinoma. Oncol Lett 7:1556-1562.

Müller H, Bracken AP, Vernell R, Moroni MC, Christians F, Grassilli E, Prosperini E, Vigo E, Oliner JD, and Helin K(2001) E2Fs regulate the expression of genes in volved in differentiation, development, proliferation, and apoptosis. Genes Dev $\mathbf{1 5}$ $267-285$

Nakamura K, Shinozuka K, and Yoshikawa N(2015) Anticancer and antimetastatic effects of cordycepin, an active component of Cordyceps sinensis. J Pharmacol Sci 127:53-56. 
Nasser MI, Masood M, Wei W, Li X, Zhou Y, Liu B, Li J, and Li X(2017) Cordycepin induces apoptosis in SGC-7901 cells through mitochondrial extrinsic phosphorylation of PI3K/Akt by generating ROS. Int J Oncol 50:911-919.

Okkenhaug K, Graupera M, and Vanhaesebroeck B(2016) Targeting PI3K in cancer: impact on tumor cells, their protective stroma, angiogenesis, and immunotherapy. Cancer Discov 6:1090-1105.

Privette Vinnedge LM, Benight NM, Wagh PK, Pease NA, Nashu MA, Serrano-Lopez J, Adams AK, Cancelas JA, Waltz SE, and Wells SI(2015) The DEK oncogene promotes cellular proliferation through paracrine Wnt signaling in Ron receptorpositive breast cancers. Oncogene 34:2325-2336.

Privette Vinnedge LM, Ho SM, Wikenheiser-Brokamp KA, and Wells SI(2012) The DEK oncogene is a target of steroid hormone receptor signaling in breast cancer. PLoS One 7:e46985.

Riveiro-Falkenbach E and Soengas MS(2010) Control of tumorigenesis and chemoresistance by the DEK oncogene. Clin Cancer Res 16:2932-2938.

Soekarman D, von Lindern M, van der Plas DC, Selleri L, Bartram CR, Martiat P, Culligan D, Padua RA, Hasper-Voogt KP, Hagemeijer A, et al.(1992) Dek-can rearrangement in translocation $(6 ; 9)(\mathrm{p} 23 ; \mathrm{q} 34)$. Leukemia 6:489-494.

Wang Y, Mo H, Gu J, Chen K, Han Z, and Liu Y(2017) Cordycepin induces apoptosis of human acute monocytic leukemia cells via downregulation of the ERK/Akt signaling pathway. Exp Ther Med 14:3067-3073.

Wise-Draper TM, Morreale RJ, Morris TA, Mintz-Cole RA, Hoskins EE, Balsitis SJ, Husseinzadeh N, Witte DP, Wikenheiser-Brokamp KA, Lambert PF, et al.(2009) DEK proto-oncogene expression interferes with the normal epithelial differentiation program. Am J Pathol 174:71-81.

Xiang J, Sun H, Su L, Liu L, Shan J, Shen J, Yang Z, Chen J, Zhong X, Ávila MA, et al.(2017) Myocyte enhancer factor 2D promotes colorectal cancer angiogenesis downstream of hypoxia-inducible factor $1 \alpha$. Cancer Lett 400:117-126.

Xu YF, Liu ZL, Pan C, Yang XQ, Ning SL, Liu HD, Guo S, Yu JM, and Zhang ZL(2019) HMGB1 correlates with angiogenesis and poor prognosis of perihilar cholangiocarcinoma via elevating VEGFR2 of vessel endothelium. Oncogene 38: 868-880.

Xu Y, Liang Z, Li C, Yang Z, and Chen L(2017) LCMR1 interacts with DEK to suppress apoptosis in lung cancer cells. Mol Med Rep 16:4159-4164.
Yang C, Zhao L, Yuan W, and Wen J(2017a) Cordycepin induces apoptotic cell death and inhibits cell migration in renal cell carcinoma via regulation of microRNA-21 and PTEN phosphatase. Biomed Res (Aligarh) 38:313-320.

Yang H, Zhang H, Ge S, Ning T, Bai M, Li J, Li S, Sun W, Deng T, Zhang L, et al.(2018) Exosome-derived miR-130a activates angiogenesis in gastric cancer by targeting C-MYB in vascular endothelial cells. Mol Ther 26:2466-2475.

Yang Y, Gao M, Lin Z, Chen L, Jin Y, Zhu G, Wang Y, and Jin T(2017b) DEK promoted EMT and angiogenesis through regulating PI3K/AKT/mTOR pathway in triple-negative breast cancer. Oncotarget 8:98708-98722.

Yokoi K, Kobayashi A, Motoyama H, Kitazawa M, Shimizu A, Notake T, Yokoyama T, Matsumura T, Takeoka M, and Miyagawa SI(2018) Survival pathway of cholangiocarcinoma via AKT/mTOR signaling to escape RAF/MEK/ERK pathway inhibition by sorafenib. Oncol Rep 39:843-850.

Zeng Y, Lian S, Li D, Lin X, Chen B, Wei H, and Yang T(2017) Anti-hepatocarcinoma effect of cordycepin against NDEA-induced hepatocellular carcinomas via the PI3K/Akt/mTOR and Nrf2/HO-1/NF-кB pathway in mice. Biomed Pharmacother 95:1868-1875

Zhang Y, Liu J, Wang S, Luo X, Li Y, Lv Z, Zhu J, Lin J, Ding L, and Ye Q(2016 The DEK oncogene activates VEGF expression and promotes tumor angiogenesis and growth in HIF-1 $\alpha$-dependent and -independent manners. Oncotarget 7: 23740-23756.

Zhou Y, Guo Z, Meng Q, Lu J, Wang N, Liu H, Liang Q, Quan Y, Wang D, and Xie $\mathrm{J}(2017)$ Cordycepin affects multiple apoptotic pathways to mediate hepatocellular carcinoma cell death. Anticancer Agents Med Chem 17:143-149.

Address correspondence to: Xiangshan Ren, Department of Pathology and Cancer Research Center, Yanbian University Medical College, No. 977, Gongyuan Road, Yanji 133002, China. E-mail: renxsh@ybu.edu.cn; or Zhenhua Lin, Department of Pathology and Cancer Research Center, Yanbian University Medical College, No. 977, Gongyuan Road, Yanji 133002, China. E-mail: zhlin720@ybu.edu.cn 